\title{
A Case Series of Intraluminal Migration Gossypiboma in Regional Hospital of Maradi
}

\author{
Ousseini Adakal, General Surgery \\ Rouga Moumouni Mohamed, General Surgery \\ Maman Bachir Abdoulaye, General Surgery
}

General Surgery Department, Regional Hospital of Maradi, Faculty of Health Sciences of Dan Dicko Dankoulodo University, Maradi, Niger

Harissou Adamou, General Surgery

Ibrahim Amadou Magagi, General Surgery

General Surgery Department, National Hospital of Zinder, Faculty of Health

Sciences, University of Zinder, Niger

Lassey James Didier, General Surgery

Rachid Sani, General Surgery

Faculty of Health Sciences, University of Niamey, Niger

Doi:10.19044/esj.2019.v15n30p331 URL:http://dx.doi.org/10.19044/esj.2019.v15n30p331

\begin{abstract}
Introduction: Textiloma or Gossypiboma is used to describe a foreign body composed of a compress (s) or surgical field (s) forgotten at an operating site. Case report: Authors reported two cases of intestinal migrant textiloma in patients all previously operated on by cesarean section. The two patients consulted for a chronic painful abdominal mass. Abdominal ultrasound showed a superficial anterior structure in favor of foreign body in the first patient. The second one concluded that there was a right ovarian cyst. The surgery discovered a greek mass in both cases. Resection of the intestinal mass was performed followed by end to end anastomosis. The enterostomy of hail mass revealed an abdominal compress in each case. Conclusion: Forgetting a compress or abdominal field during surgery is a tragedy for the surgeon not only because of its forensic aspect but also because of the suffering endured by the patient. Indeed, actions trying to prevent this type of error are implemented in most developed countries; it is time for each hospital to adopt preventive measures, even if just only the compresses counting during the procedure.
\end{abstract}

Keywords: Textiloma, Gossypiboma, Foreign body, Maradi, Niger 


\section{Introduction}

Textiloma, commonly called Gossypiboma by Anglo-Saxons, is used to describe a foreign body composed of a compress (s) or surgical field (s) forgotten at an operating site. [Margonis, 2016; Faraj, 2013]. It is an omission in a part of the human body of a foreign body during a surgical procedure. The various writings agree that this condition is rare, even if its medico-legal repercussions should not be minimized [Erguibi, 2015; Hammoud,2001]. Its manifestations depend on the biological evolution of the foreign body, which can have two aspects: inflammatory with the formation of a granuloma that can mimic several pathologies leading to diagnostic and exudative wandering leading to an abscess or fistulization [Gawande, 2003]. Textiloma migration is a rare phenomenon. We report two cases of intestinal migrant textiloma managed at the Regional Hospital of Maradi and we will review the literature.

\section{Case 1:}

Authors report a case of a thirty (30) years old patient, seventh gesture, seven anterior parities and five living children, with a past medical history of three surgical procedures. The first was for appendicitis, the two others for cesarean sections. The last one was performed three years ago for a suspicion of disunion. She was admitted for exploratory laparotomy for an abdominal mass associated with abdominal pain evolving over the past 3 years. It should be noted that this pain started about three weeks after the last cesarean section. This was combined with transit disorders and constipation of an episodic nature. The clinical examination showed a patient in good general condition, blood pressure (BP): $13 / 8 \mathrm{~cm} \mathrm{Hg}$, Temperature: $37^{\circ} \mathrm{C}$, pulse: 76 pulse/ $\mathrm{mn}$, Respiratory frequency: 21 cycles/ $\mathrm{mn}$, weight / 64 Kilogrammes. The abdominal examination showed on inspection, 3 incision scars: Mac Burney, Pfannential and median subumbilical incisions; a hard, painful and mobile periombilical mass was palpated in relation to the superficial and deep plane. There was no hepato-splenomegaly or declive matte. The rest of the examination was without particularity. The biological check-up was normal. Abdominal ultrasound showed a superficial anterior structure that appeared flat with a thickness of $7 \mathrm{~mm}$ in favor of a foreign body. [Figure1]. 


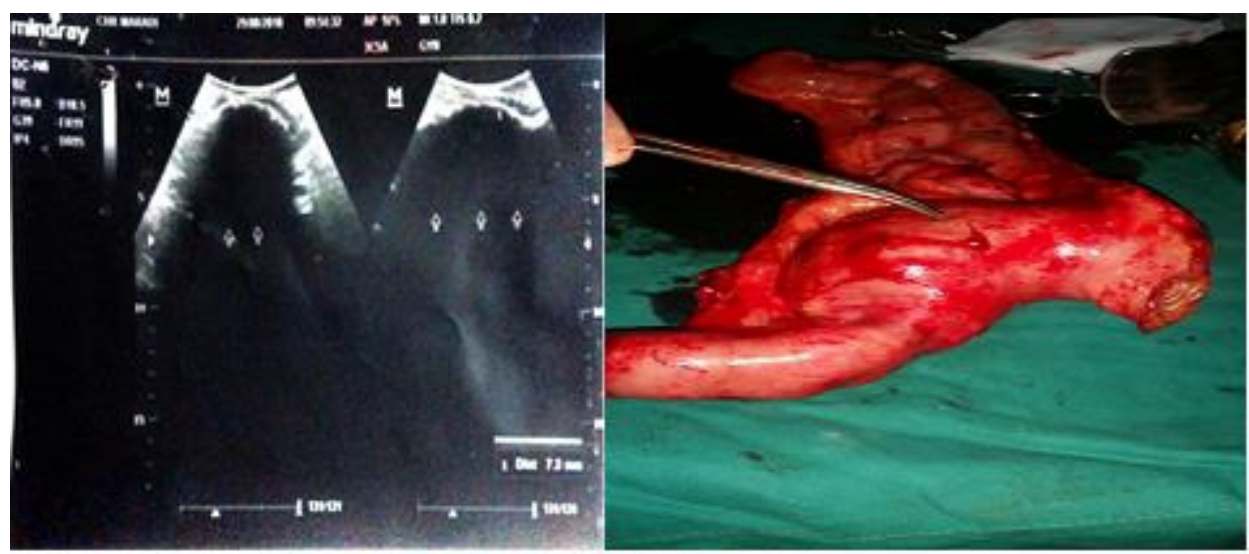

Figure1 : indrect ultrasond signsind cating foreign body

Figure 2: hail mass resection piece

The surgery discovered a small intestine mass about $130 \mathrm{~cm}$ from the ileo caecal junction. Resection of the mass was performed followed by end to end anastomosis [Figure 2]. The enterostomy of the removed mass revealed an average abdominal field of $30 \mathrm{~cm} \times 45 \mathrm{~cm}$ digested and molded into the small intestine [Figure 3].

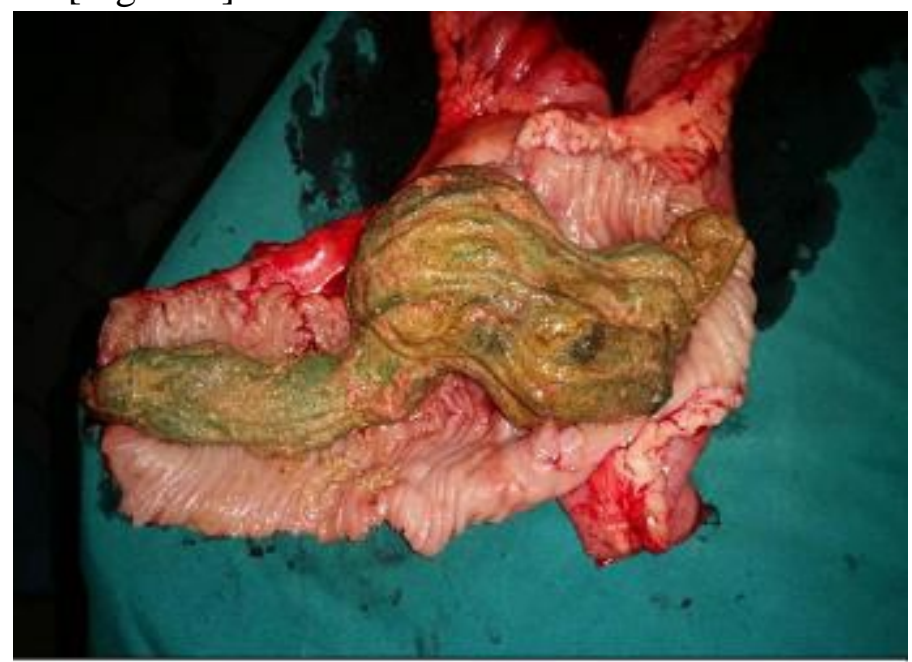

Figure 3: Enterostomy showing an abdominal compress

The postoperative outcome was simple and the patient left the hospital at the sixth day after surgery.

\section{Case 2:}

Authors report the case of N.A, a 29 years old lady, 5th Gesture and 5 previous parities, 2 living children and 3 deceased. She consulted for pelvic pain located in right iliac fossa that has been progressing for a few weeks with 
diarrhoea and vomiting. Her surgical history included a cesarean section for early dystocia 9 years ago and a hysterectomy following an undocumented delivery 4 years ago. The clinical examination found a good general condition, a flexible, slightly sensitive abdomen with the presence of a hard periombilical mass, irregular and mobile in relation to the deep plane. The abdominal ultrasound objectified a right ovarian cyst of 54 x $52 \mathrm{~mm}$, reason of surgical indication of a right ovarian cystectomy. At the abdominal opening revealed a mass encompassing several portions of the small intestine up to about $60 \mathrm{~cm}$ from the Ileo-caecal junction. A monoblock resection was performed removing the mass and the portions of the small intestine involved (about 80 $\mathrm{cm}$ of small intestine) followed by an end to end anastomosis. The postoperative outcome was uneventful and the patient was exited from the hospital on the fifth day post operation.

The enterostomy of the mass allowed the discovery of abdominal compresses of $35 \mathrm{~cm} \times 40 \mathrm{~cm}$ digested and molded in the small intestine [Figure 4].

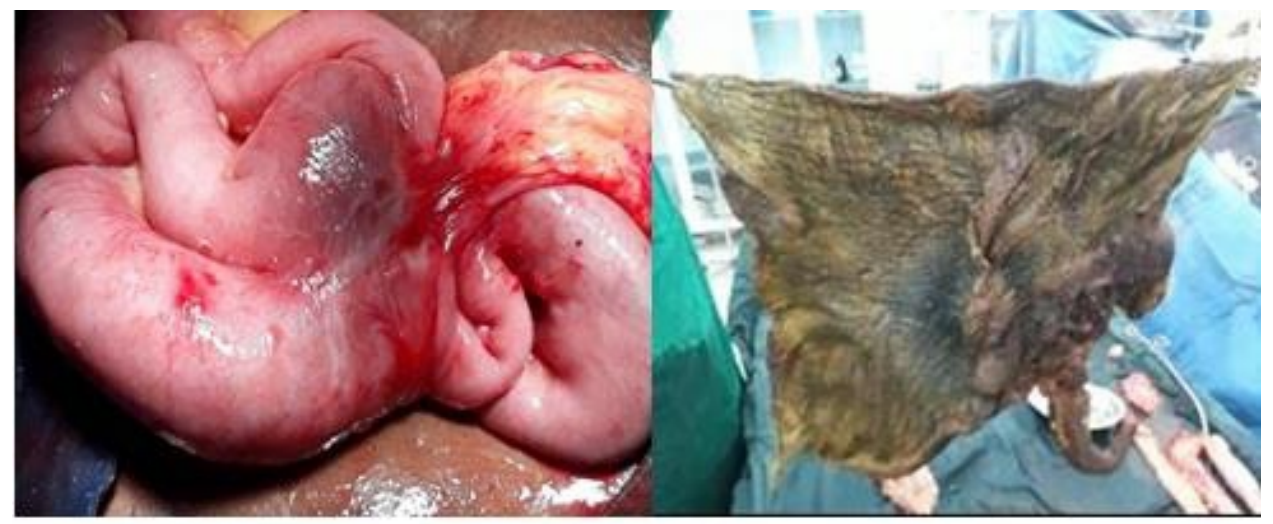

Figure 4: intraoperative view of the hail mass

Figure 5: abdominal compress after enterostomy

\section{Discussion}

Gossypiboma is a term derived from "gossypium" (cotton in Latin) and "boma" (hiding place in Swahili) [Kassi, 2018]. As surgery progressed, the use of synthetic materials took precedence over the use of cotton, which was once used to ensure hemostasis, hence the term textilome (derived from textiles and the suffix "ome" which means growth) [Nassar, 2004]. Forgetting an object, a compress, surgical equipment or any other preoperative element in a patient's body is a dramatic incident or accident not only for the patient, but also for the surgeon. Despite the preventive measures developed to reduce the risks, these accidents remain and continue to be experienced by surgical teams. Although the actual incidence is underestimated due to the forensic 
nature of the condition, there are figures in the literature ranging from $1 / 1,000$ to 1/10,000. [Nassar, 2004; Arsalane, 2005]. The risk of forgetting a foreign body is linked to certain particular situations, as Gawandé points out that uncontrolled bleeding, obesity, and lack of compress count are all factors that increase this risk [Gawande, 2003]. All forms of surgery can be involved with various localizations: cranial [Nassar, 2004], thoracic [Arsalane, 2005], urological [Doh, 2017], gynaecological [Osman, 2013]. The most frequent localization is abdominal-pelvic, which can have several clinical aspects, linked to the biological evolution of the foreign body within the abdomen. This natural biological evolution is reflected in the pathophysiological expression of textile fibres, which lead to inflammatory reaction with exudation from 24 hours onwards, followed by the formation of granulation tissue (8th day); fibrosis is organised from the 13th day onwards. This evolution explains, in the absence of infection, the possibility of encysting or even calcifications with a sometimes long tolerance [Faraj, 2013; Erguibi, 2015]. Late evolution of the textilome, error or diagnostic delay often lead to migration of the compress or field through an organ [Kierman, 2008]. The case we report is a migration of an abdominal field (tetra compress) from the large peritoneal cavity to the intestinal lumen [Figure 3 and 4]. This type of complication is rare. It is done from the site of origin due to an inflammatory exudative reaction, with the formation of internal or external chronic abscesses or fistulas [Faraj, 2013; Hammoud, 2001]. In case of migration into the intestinal lumen, the loop closes after a complete migration of the compress and peristaltic activity moves it to the terminal ileum where it stagnates, often resulting in intestinal obstruction [Faraj, 2013]. In our observation, intraluminal migration is identical to a few very rare cases described in the literature[Margonis,2016; Gawande, 2003; Kassi, 2018; Kierman, 2008; Lv, 2014]. The foreign body seems to mold itself to the intestinal lumen but without obstructing it, intermittently causing abdominal pain, which is one of the reasons for repeated consultations in our two patients. The diagnosis is not easy except in chronic situations where the clinical examination may find a palpable mass or even fistulization to the skin or the externalization of the foreign body through the anus [Faraj, 2013]. Indirect signs may lead to the search for a foreign body in the event of previous surgery or signs of sub-occlusion. It is in this capacity that some reviews find their interests. Thus, the diagnosis can be easily made if it is a radiolabelled compress. In our context, compresses are neither marked nor counted during surgical procedures. The use of abdominal fields made by hand and not radio-opaque makes diagnosis by standard radiography even more difficult. The ultrasound, the routine examination most frequently encountered and requested in our two patients, can find three aspects: an echogenic formation with posterior shadow cone, the posterior shadow cone being secondary to the stopping of the ultrasonic beam by the textile fragment; 
a well-defined cystic formation containing hyperechoic, snake-like and scratched internal structures, the latter corresponding to the forgotten textile; non-specific aspects, hypoechogenic or simple cystic formation [Hammoud, 2001; Mercier, 2016]. The second aspect is most frequently reported in the literature and is the one described in our observation. Textiloma management remains surgical and any diagnostic or therapeutic delay can be fatal in some locations. This is the case described by Osman who described a uterine carcinosarcoma revealed 9 years after surgery. This shows the importance of many safety practices proposed to prevent or reduce the occurrence of textilomes. These are the counting of compresses, the use of radiolabelled compresses, routine radiography after any major intervention and new recommendations such as barcodes, [Hammoud, 2001; Osman, 2013]. These preventive practices are not common in our context, hence the interest for any surgeon to be vigilant to avoid serious complications for the patient on the one hand and the medico-legal consequences raised by an intra-abdominal foreign body on the other [Uluçay, 2010].

\section{Conclusion}

Forgetting a compress or abdominal field during surgery is a tragedy for the surgeon and all surgical team, not only because of its forensic aspect but also because of the suffering endured by the patient. Indeed, procedures of preventing this type of error are in place in most developed countries, it is time for each hospital to adopt a preventive measure, if only the counting of compresses

\section{References:}

1. Margonis E, Vasdeki D, Diamantis A, Koukoulis G, Christodoulidis G, Tepetes K. (2016) Intestinal Obstruction and Ileocolic Fistula due to Intraluminal Migration of a Gossypiboma. Case Rep Surg. 2016:1-4.

2. Faraj PA, Berbich PA, Lazrak PB, Chkili PT, Alaoui PMT, Belmahi PA. (2013) Les Textilomes intrapéritonéaux à propos de 2 cas avec revue de la littérature. Thèse $\mathrm{N}^{\circ} 75$. Université Mohammed V-Souissi Faculté de Médecine et de Pharmacie - Rabat. Année: 2013: 170.

3. Erguibi D, Hassan R, Ajbal M, Kadiri B. (2015). Textilome abdominal, à propos d'un cas. Pan Afr Med J. 21:244 doi:10.11604/pamj.2015.21.244.7194, Disponible sur: http://www.panafrican-med-journal.com/content/article/21/244/full/

4. Hammoud D, Ammouri N, Rouhana G, Saad H, Husseini H, Sleiman CA. (2001) Aspects Radiologiques Des Textilomes. J Radiol. 82: 9136. 
5. Gawande AA, Studdert DM, Orav EJ, Brennan TA, Zinner MJ. (2003) Risk Factors for Retained Instruments and Sponges after Surgery. N Engl J Med. 348(3):229-35.

6. Kassi ABF, Yenon KS, Koffi EM. (2018) A transmural migration of a gossypiboma in the right colon responsible for a mass which mimicked an abscessed colonic tumor: A case report. Int J Surg Case Rep. 51:228-30.

7. Nassar I, Mahi M, Semlali S, El Quessar A, Chakir N, El Hassani MR. (2004) Textilome Intracrânien. J Neuroradiol. 31(2):148-9.

8. Arsalane A, Kabiri H, Zidane F, Maslout A, Benosman A. (2005) Textilomes thoraciques. Rev Pneumol Clin. 61(4):243-6.

9. Doh K. (2017) Un cas de textilome renal simulant une tumeur de découverte anatomopathologique. African Journal of Urology. 23: 364-367

10. Osman K, Abdellaoui B, Weyl B, Levêque J. (2013) Carcinosarcome utérin secondaire à un textilome vaginal : à propos d'un cas et revue de la littérature. J Gynécologie Obstétrique Biol Reprod. 42(1):91-4.

11. Kiernan F, Joyce M, Byrnes CK, O'Grady H, Keane FBV, Neary P. (2008) Gossypiboma: a case report and review of the literature. Ir J Med Sci. 177(4):389-91.

12. Lv Y-X, Yu C-C, Tung C-F, Wu C-C. (2014) Intractable duodenal ulcer caused by transmural migration of gossypiboma into the duodenum - a case report and literature review. BMC Surg. 14(1):36.

13. Mercier M, Noailles T, Sali E, Carret P, Duvauferrier R, Rouvillain JL. (2016) What type of imaging work-up will help to confirm the diagnosis of gossypiboma in the limb? Review of literature. Orthop Traumatol Surg Res. 102(6):795-800.

14. Uluçay T, Dizdar MG, SunayYavuz M, Aşırdizer M. (2010) The importance of medico-legal evaluation in a case with intraabdominal gossypiboma. Forensic Sci Int. 198 (1-3):e15-8. 